\title{
NAVEGACIÓN DE ROBOTS MÓVILES EN ENTORNOS CON DISCONTINUIDADES: UNA REVISIÓN
}

\author{
Luis Fernando Ortiz Arroyave ${ }^{1}$, Mauricio Vásquez Carvajal ${ }^{2}$, Nelson David Muñoz Ceballos ${ }^{3}$ \\ ${ }^{1}$ Ing. Electrónico, Estudiante Maestría en Automatización y Control Industrial del Instituto Tecnológico \\ Metropolitano- ITM, Instructor Servicio Nacional de Aprendizaje SENA. \\ ${ }^{2}$ M.Sc. en Automatización y Control Industrial, Docente Instituto Tecnológico Metropolitano- ITM. \\ ${ }^{3}$ M.Sc. en Automatización y Control Industrial, Docente, Politécnico Colombiano Jaime Isaza Cadavid. \\ Medellín - Colombia \\ Email: Ifortiz7@gmail.com
}

\begin{abstract}
RESUMEN
Se realizó una revisión del estado del arte en torno a la navegación de robots móviles con ruedas, donde particularmente se analizan algunas técnicas usadas en el tratamiento de los hitos, particularidades o discontinuidades que se puedan encontrar en una posible ruta óptima, haciendo principal énfasis en la navegación en entornos con pasillos y puertas, dado que son elementos importantes cuando se habla de navegación en entornos interiores como casa, edificios, fábricas, etc., se analizan temas desde la adquisición de la señal, estrategias para el procesamiento de la información y las respuestas obtenidas.
\end{abstract}

Palabras Claves: Robótica móvil, Navegación autónoma, discontinuidades, Procesamiento de información.

Recibido: 18 de septiembre de 2017. Aceptado: 13 de septiembre de 2018

Received: September 18th, $2017 . \quad$ Accepted: September 13th, 2018

\begin{abstract}
A review about wheeled mobile robots navigation is made, where some techniques used in the treatment of milestones, particularities or discontinuities that can be found in a possible optimal route will be analyzed, with main emphasis on navigation in environments with corridors and doors, given that they are an important item when talking about navigation in indoor environments such as houses, buildings, factories, etc., in this topics will be analyzed from the acquisition of the signal, strategies for processing information and responses obtained.
\end{abstract}

Keywords: Mobile robotics, Autonomous navigation, discontinuities, Information processing.

Cómo citar este artículo: L. F. Ortiz Arroyave, M. Vásquez Carvajal, N. D. Muñoz Ceballos, "Navegación de robots móviles en entornos con discontinuidades: una revisión", Revista Politécnica, vol. 14, no. 27 pp.103115, 2018. https://doi.org/10.33571/rpolitec.v14n27a10 


\section{INTRODUCCIÓN}

La robótica es una de las áreas del conocimiento que más desarrollo ha tenido durante los últimos años. Un robot se entiende como "un sistema electromecánico, dotado de sensores y actuadores para realizar actividades similares a las realizadas por seres vivos, con un sistema de control y capacidad de decisión que le permite realizar tareas automáticas o autónomas, dependiendo de su grado de versatilidad y complejidad" [1].

Dentro de los 2 campos entre los cuales se ha enfocado la robótica, cabe destacar el área industrial centrada en robots manipuladores y un segundo bloque en el que sobresalen los robots móviles o de servicios [2, 3], donde este último, se entiende como un sistema con capacidad para desplazarse de un sitio a otro de forma telecontrolada o autónoma con el objeto de realizar una tarea para la cual ha sido diseñado [4], para ello se hace importante resaltar que tan relevante es la autonomía, la cual busca que los robots lleguen a ser sistemas autónomos completos que puedan operar eficientemente en entornos complejos sin necesidad de estar constantemente guiados y controlados por operadores humanos [5], ademas que las plataformas puedan desplazarse sin problemas cuando surjan obstaculos en su recorrido, teniendo la habilidad de cambiar o seguir su rumbo libre de colisiones [6], lo cual podra ayudar a los seres humanos en la toma de decisiones rapidas o delicadas como en el tema de los vehiculos automoviles, las sillas de ruedas para personas discapacitadas [7, 8, 9] o hasta en las tareas de búsqueda y asistencia humana donde se pueda ver afectada la vida de rescatistas [10]

Otro aspecto importante a la hora de analizar una plataforma robótica móvil es la sensorica que disponga para poder obtener la información del medio ambiente, como es capaz de procesar los datos adquiridos y a partir de dicho análisis, tomar una decisión correcta para actuar, navegando de forma segura a través del entorno [11, 12], para algunas investigaciones, se puede evidenciar la existencia de dos grandes grupos de algoritmos de navegación [13]. Por un lado, están los algoritmos basados en información completa o mapas del entorno donde van a moverse, por otro se encuentran los algoritmos basados en información incompleta, donde en la mayoría de los casos los robots no dispondrán de un mapa del entorno sobre el que se van a mover [13], de este caso se pueden encontrar varias aplicaciones para navegación autónoma en entornos desconocidos, tales como:

Seguridad y vigilancia, Silla de ruedas autónomas, Desactivación de explosivos, Agricultura, Exploración de tuberías, Exploración planetaria, entre otros.

Para las aplicaciones mencionadas anteriormente, un robot debe realizar diferentes actividades $u$ operaciones internamente, esto con el fin de poder realizar sus tareas en el mundo real [22] y cuando todas las variables se correlacionen entre la dinámica del robot y su entorno aparecerá cierto grado de incertidumbre, además, se debe tener en cuenta que a menudo la planificación se considerada como un acto fuera de línea dados los tiempos que hay entre preparar el movimiento y ejecutarlo [23], es decir, estos problemas pueden invalidar el plan antes de que el robot sea capaz de tomar una decisión que sea apropiada o pertinente. Estos problemas han sido históricamente abordados desde dos puntos de vista diferentes, que dieron lugar a dos líneas de investigación paralelas: métodos de replanificación (parciales) y técnicas de evasión de obstáculos [22]. Para otros autores, dichas soluciones se han presentado de otra forma, de acuerdo a ella han surgido 4 subdivisiones en la robótica móvil, siendo estos: en primer lugar, el sistema deliberativo o jerárquico $[18,24,25]$ donde se requiere de una planificación previa para la realización de una acción, en un segundo escalón se aparecen los sistemas reactivos, donde solamente se usan la localización o posición donde se encuentren y a partir de ello cuáles serán las acciones a tomar para elaborar una tarea [26, 27, 28], pero preveen la ventaja de extensibilidad, respuesta en tiempo real y bajo costo computacional [29]. A partir de la combinación de los 2 sistemas mencionados anteriormente aparecen los métodos híbridos, donde el sistema deliberativo está por encima del sistema reactivo, el primero es quien planea y el segundo se encarga de la forma [30, 31, 32] y por ultimo están los sistemas con aprendizaje, donde aparece un nuevo ítem para tener en cuenta como es el aprendizaje [33].

Además de lo anterior, es importante resaltar que los robots deben desplazarse en 2 tipos diferentes de entornos, siendo estos en exteriores e interiores, en cada uno de ellos se presentan diferentes tipos de discontinuidades donde las plataformas deben 
afrontar ambientes locales distintos que influyen durante cada desplazamiento [34].

En este artículo se analizan algunos temas referentes a la navegación autónoma de robots móviles con ruedas, especialmente la trayectoria del vehículo a través de algunas particularidades o discontinuidades que se puedan encontrar en una posible ruta optima durante el cumplimiento de una tarea. Se hace principal énfasis en pasillos y puertas. Para lo anterior el artículo estará distribuido en 3 bloques: En la primer parte, se trataran algunos trabajos o técnicas que se han realizado en lo referente a pasillos y puertas. En el segundo de los bloques se relacionan métodos que hay en cada parte de un robot, como en la adquisición de las señales capturadas por los sensores, el procesamiento de estas señales y las decisiones que se toman a partir de ellas con el fin de evadir algunas de dichas discontinuidades que se puedan presentar para el cumplimiento de la labor asignada. En el tercero de los segmentos se muestran los resultados de la discusión presentada en el literal anterior, junto con las tendencias que se trabajaran en esta área. Finalmente se presentan las conclusiones.

\section{MATERIALES Y METODOS}

Normalmente los robots móviles deben ser capaces de moverse en un entorno con cierto grado de autonomía, para ello la navegación autónoma debe estar asociada a la disponibilidad de sensores externos que capturen información del entorno a través de imágenes visuales o mediciones de distancia o proximidad. Los sensores más comunes son los sensores de distancia (ultrasónicos, infrarrojos, láser, cámaras, etc.) capaces de detectar obstáculos y de medir la distancia a los obstáculos cercanos a la trayectoria del robot. Cuando los robots autónomos avanzados navegan dentro de ambientes interiores (edificios industriales o civiles), tienen que ser dotados de la capacidad de moverse por los pasillos, de seguir las paredes, de dar vuelta a las esquinas y de entrar en áreas abiertas de las habitaciones [35], a estos últimos se les denomina hitos o discontinuidades en la navegación de un robot.

Para trabajar algunos de estos hitos, se han realizado múltiples investigaciones, en esta revisión se le brinda un enfoque especial a la navegación a través de pasillos y como tratar los movimientos a través de una puerta, Inicialmente se hablara sobre que estrategias se han trabajado a nivel de la movilidad de una plataforma robótica a través de un pasillo, teniendo en cuenta que han sido varias las formas en que se han tratado.

Se han propuesto varias soluciones para la navegación en pasillos, por ejemplo, en [23] se presentan estrategias para robots que manejan comportamientos reactivos, la estrategia que se emplea generalmente es la técnica denominada seguimiento de pared, en cualquiera de sus 2 versiones, ya sea derecha o izquierda, de acuerdo al caso se busca llevar una distancia imaginaria desde el robot hasta la pared [35] y a nivel estadístico en lo que se denomina características topológicas de alto nivel (entre ellos los pasillos) se usa un enfoque bayesiano [36], donde se trata de representar la densidad de probabilidad a través de algunos algoritmos tales como condensation [37] o Rechazo [38].

Otros autores han propuesto abordar el problema de navegación a través de corredores, haciendo uso de algunos algoritmos de control, para ello han realizado la clasificación en 3 niveles.

El primero, basado en visión artificial, el procesamiento de imágenes se emplea para detectar líneas de perspectiva para guiar al robot a lo largo del eje central del corredor [39]; otros investigadores aprovechan las líneas de perspectiva de las cámaras para trabajar con referencia al techo, donde se usan para la localización de vehículos en los corredores [38]. En [40], se manejan dos cámaras laterales, montadas en el robot, las cuales se destinan para obtener de ambas imágenes la velocidad aparente, estas se comparan y del resultado que se obtiene se puede controlar la velocidad del robot. En [41,42], se utiliza una cámara para guiar el robot a lo largo del eje del corredor o para seguir una pared, utilizando el cálculo del flujo óptico y sus derivadas temporales.

En un segundo nivel se basan en odometría y sensores para detección de obstáculos, para ello se plantean algunos trabajos tales como: en [22] donde se desarrolla un algoritmo de control globalmente estable para el seguimiento de la pared basado en codificadores incrementales y un sensor sonar, en [13] se desarrolla un algoritmo de control para guiar a un robot móvil a lo largo de un corredor o para permitir que el robot siga una 
pared, el algoritmo se basa en mediciones de distancia suministradas por sensores sonar, está diseñado para ser asintóticamente estable y evitar la saturación de los actuadores del robot o para aplicaciones más específicas como en un Invernadero, en donde el recorrido óptimo es en zigzag y el tiempo de operación, los obstáculos o las plantaciones son detectadas a partir de sensores infrarrojos o ultrasonidos [43].

En el tercer nivel se encuentran los sistemas mixtos que son aquellos que trabajan en combinación de la odometría e imágenes capturadas por medio de visión artificial para estimar la posición de obstáculos, acá se encuentran investigaciones tales como en [36], allí se utilizan 2 controladores, el primer controlador utiliza el flujo óptico medido a partir de las paredes laterales del corredor para generar un comando de velocidad angular, el segundo esquema utiliza la información de distancia obtenida con sensores de ultrasonidos para el cálculo de la posición del robot y la orientación con respecto a la línea central del pasillo, se emplea un filtro de información descentralizada que minimiza el nivel de incertidumbre en ambos controladores. Esta incertidumbre se evalúa en términos de la detección de errores y las condiciones del entorno por medio de una función de covarianza para cada controlador.

Fuera de las estrategias aplicadas, aparece un ítem aún más importante dentro de este análisis, el cual es, el área de navegación donde se están aplicando las estrategias mencionadas y otras, entre ellas están:

- Travesía, donde sobresalen los trabajos en navegación autónoma en sillas de ruedas [14, 44], algunos de estos proyectos son: Proyecto Tao [22], NavChair [23], proyecto europeo FP7 Radhar [45] y el proyecto SYSSIASS [46], en algunos casos, estos proyectos también trabajan sobre el paso en puertas estrechas [47].

- En áreas de agricultura, en labores de riego de insecticida en invernaderos, donde la salud de quienes manipulan estos químicos, podría verse afectada [16, 17], o en la agricultura de precisión $[18,48]$.

- Patrullaje: donde se busca identificar intrusos, posibles focos de fuego, etc. [10, $11,30]$.
- Búsqueda y rescate de personas [49]

- Robot guía de turistas en museos, comercios, etc. [50]

- Desactivación de artefactos. explosivos [15, 51].

- Supervisión [19].

- Exploración de tuberías [20].

- Exploración planetaria [21], entre otros.

La navegación de plataformas móviles a través de puertas es un aspecto relevante cuando se trabaja en ambientes interiores, dado que es crucial decidir si se ingresa a dicho espacio o se busca otra ruta para poder cumplir la tarea asignada, además de lo anterior también es relevante identificar el estado de la puerta ya sea abierta, medio abierta o cerrada [52]. Las técnicas para la detección de puertas en espacios interiores se pueden agrupar en métodos no visuales $y$ visuales. Para los primeros se han combinado sensores de ultrasonido y video para localizar puertas, pero debido a ciertos materiales con los cuales son fabricadas las puertas el coeficiente de reflexión de las ondas sonoras puede distorsionarse, causando imprecisiones [53]. En [54] proveen una silla de ruedas de dispositivos sonar para ayudar a personas con dificultades de visión o de movilidad reducida para localizar puertas abiertas, teniendo algunas limitaciones cuando hay varias personas con el mismo dispositivos en el mismo entorno, dado que se pueden interferir entre sí. En el segundo grupo se han elaborado investigaciones que trabajan en $2 \mathrm{D}$ y 3D, por ejemplo en [55] se trabaja con un láser en 3D a color el cual proporciona la geometría de la puerta (coordenadas xyz) y el color, permitiendo analizar si la puerta está abierta o cerrada. En otra investigación se hizo uso de la cámara de profundidad Microsoft Kinect la cual captura algunas imágenes que son comparadas con otras predeterminadas, para detectar las puertas son comparadas las dimensiones [56]. En [57] se trabaja con visión en donde para la detección de puertas se analizan características tales como colores, tamaños, formas, orientaciones y texturas

\subsection{Estrategias de adquisición de Información de los Sensores}

Es importante resaltar que la elección de los transductores a usar está sujeta a las características del problema a tratar, dado que en robótica, los sensores son los elementos que le permiten extraer información correcta de su 
entorno. Además de ello hay que conocer las diferencias que hay entre algunos tipos de sensores:

- Visuales (un haz de luz se refleja sobre los objetos) y no visuales (las señales que se reciben son sonoras, inerciales, otras) [52].

- Internos muestran la orientación, posición del robot y externos que son los que perciben el entorno de la plataforma.

- Activos, los cuales emiten energía al entorno, mientras los pasivos son quienes la reciben [58].

La mayoría de los datos provenientes de los sensores presentan problemas tales como ruido, imprecisiones en sus medidas, etc. lo cual genera en algunas ocasiones información incompleta del entorno [59], para poder dar solución a estos y otros problemas han aparecido diversos métodos, entre ellos están el uso de redes neuronales que se usan para dar confiabilidad a los datos, también son capaces de identificar los estados y las transiciones entre los estados [11, 60]. El uso de métodos difusos proporciona una tolerancia propia a la incertidumbre en los datos [61] y permite la combinación de la información proveniente de diversos sensores de una plataforma robótica [62], donde las reglas se derivan de detalladas observaciones del sistema.

Dentro de los sistemas estocásticos, la técnica denominada filtro de Kalman, es el método más utilizado para la integración de datos de múltiples sensores [63], dado que permite obtener una estimación de mínima varianza del estado de la variable que se está analizando [64], una variación de este sistema es el filtro de Kalman extendido, el cual posibilita trabajar con sistemas no lineales, donde se busca obtener una aproximación más certera de las medidas de los sensores $[65,66]$. Otro de los filtros que es utilizado es el llamado filtro de partículas, el cual permite resolver dificultades de incertidumbre y no linealidad en las medidas obtenidas [67]; para otras investigaciones se hizo uso de estrategias de modelos probabilísticos, dado que se puede incorporar la incertidumbre de la medida que se ha tomado [68], permitiendo calcular una distribución de probabilidad sobre lo que tal vez fuera el caso en el mundo real, en lugar de generar un único valor.

\subsection{Estrategias de Procesamiento de Información y toma de decisiones}

En cuanto al uso de inteligencia artificial se pueden encontrar nuevamente las redes neuronales las cuales tienen diversas aplicaciones en esta área, por ejemplo la identificación de hitos como paredes y pasillos [69], dado que permiten la generalización de conocimiento y el ajuste de sus salidas a muchas entradas, incluso cuando algunas entradas no se le enseño explícitamente a la red [60]. Los sistemas difusos también resultan ser beneficiosos en lo referente a la navegación autónoma, dado que permiten variar la velocidad del robot móvil, en función de las entradas de control, previamente de la decisión que sea tomada por el módulo de evasión de obstáculos [69].

Otro técnica que trabaja los pasillos es la robótica semántica y en especial una de sus herramientas como las máquinas de soporte vectorial, donde se utiliza la información propia de los sensores de distancia y además de ello todas las posibles situaciones que sean contempladas como entradas para el entrenamiento, así se logra una alta proporción de clasificaciones, allí los datos obtenidos son convertidos en representaciones geométricas simples [70]

Para el uso de aplicaciones probabilísticas en el control de navegación autónoma se considera la situación actual del entorno y una o varias rutas proyectadas [47], además son muy precisos cuando se trabaja en entornos muy grandes y no se cuenta con un dispositivo de posicionamiento global, tal como el GPS [71]. Uno de los métodos que se ha usado es el de Procesos de decisión de Markov parcialmente observables (POMDPs), el cual funciona de manera óptima en condiciones de incertidumbre en tareas de decisión secuencial [11].

Cuando lo que se busca es una construcción geométrica que permita elaborar una partición o un esquema del plano euclidiano o dibujar el contorno interior de una edificación, los diagramas de Voronoi se encargan de codificar los datos de cercanía entre componentes, para el área de enfoque que se analiza, es observar la distancia entre el robot y los obstáculos y como estrategia se busca ampliar la distancia entre ambos [48, 72]. Los grafos de visibilidad, consiste en la escogencia de 2 puntos del escenario, y estos tienen que unirse mediante una línea recta sin que exista obstáculo alguno, escogiendo como nodo inicial el 
punto inicial del recorrido y nodo final la meta del robot [43].

Las Maquinas de estado finito (FSM) permiten describir el sistema fácilmente como una secuencia de estados, teniendo en cuenta: entradas (sensores) que permite cambiar de un estado (situación) a otro, y también definir para cada estado, una acción específica (acción motor). Así que para cada cambio de estado y el estado, el robot es capaz de reaccionar adecuadamente [60], cuando se habla de estado, se hace referencia a cada una de las posibles direcciones que pudiese tomar la plataforma. Este método también es aplicable en laberintos, sobre todo en cuanto a las órdenes de control, por ejemplo en el caso en que la plataforma se encuentra moviéndose a través de un pasillo y en algún instante el sensor derecho ya indica que no hay muro, entonces ya se pasaría a otro estado como por ejemplo girar esquina [73]

Para aquellos casos en donde se trabaja con navegación basada en comportamientos, donde muchos de los investigadores tratan un comportamiento como un conjunto de trayectorias compuestas, cuyo fin es el de expresar el control del robot. Una vez que se especifica, se puede ejecutar tantas veces como sea necesario. Los comportamientos pueden ser expresados a nivel de motor, habilidad o tarea [74]. Aparecen aplicaciones a nivel de comportamientos reactivos con los cuales se pueden encontrar algunos eventos como seguimiento de línea, seguimiento de pared derecha, seguimiento de pared Izquierda, bifurcaciones y repulsión de objetos [75], estos tienen algunas características [45, 29], tales como: la percepción y la acción están estrechamente relacionadas, los comportamientos se definen a partir de los objetivos para los cuales se construye la plataforma y comportamientos complejos surgen de la combinación de comportamientos individuales más simples. Para sistemas híbridos, se puede resaltar que se destacan 2 métodos de control por comportamientos, siendo estos el competitivo [46] y el cooperativo [76]. Para este tipo de estrategia, se debe tener en cuenta que cuanto mejor sea el robot, mejor se ejecutaran los comportamientos, por ende estos comportamientos tienden a ser robustos, eficientes y adaptables a diferentes situaciones [74].
La técnica de campos potenciales, basada netamente en comportamientos reactivos, se enfoca en la planificación local en entornos desconocidos [77], en el que la plataforma es considerada como una partícula que está influenciada por un campo potencial artificial, este consta de un potencial que lo atrae hacia la meta y un potencial que hace que el robot se aleje de los obstáculos, donde el potencial resultante, se obtiene de la suma vectorial de ambos potenciales [43].

\subsection{Detección de obstáculos}

A la hora de navegar, existen muchas formas y tipos de objetos que pueden obstruir la navegación de la plataforma, cuando este trata de cumplir la tarea asignada, allí es donde se hace necesario rodearlos o pasar a través de ellos como en el caso de pasillos o puertas [6, 78], para ello se puede decir que dentro de la robótica móvil, se han desarrollado varias técnicas para dar una solución de detección de obstáculos, de ellas han sido patentadas estrategias tales como seguimiento de trayectorias con sensores para el nivelado de superficies [80], evasión de obstáculos empleando dispositivos de contacto [81], técnicas láser [82], generación de mapas de contorno [83], manejo de sensores infrarrojos [84], uso de cámaras [85] y técnicas de procesamiento digital de imágenes [39, $38,15]$, y elementos RGB-D [66] como el Kinect [9, $56]$, etc.

En la tabla 1 se resumen varios artículos de interés referente a discontinuidades en la robótica móvil. 


\begin{tabular}{|c|c|c|c|}
\hline Articulo & Temática trabajada & $\begin{array}{c}\text { Hito o } \\
\text { discontinuidad }\end{array}$ & Aspectos a mejorar \\
\hline $\begin{array}{l}\text { Guía de Personas con } \\
\text { Robots Móviles } \\
\text { Basado en la } \\
\text { Interacción Humano- } \\
\text { Robot. [50] }\end{array}$ & $\begin{array}{l}\text { Se presenta el desarrollo e } \\
\text { implementación de una nueva } \\
\text { técnica de interacción humano- } \\
\text { robot para la guía de personas } \\
\text { a través de plataformas móviles } \\
\text { con ruedas con el fin de facilitar } \\
\text { la integración de los robots en } \\
\text { la sociedad. } \\
\text { La técnica de interacción } \\
\text { propuesta consiste en un } \\
\text { sistema de control que regule } \\
\text { la velocidad del robot durante } \\
\text { el recorrido tomando en cuenta } \\
\text { la velocidad estimada del } \\
\text { usuario en tiempo real }\end{array}$ & \begin{tabular}{|l|} 
Pasillos en \\
laboratorios, \\
museos, \\
hospitales, \\
otros
\end{tabular} & \\
\hline $\begin{array}{l}\text { Door Detection in 3D } \\
\text { Colored Laser Scans } \\
\text { for Autonomous } \\
\text { Indoor Navigation [55] }\end{array}$ & $\begin{array}{l}\text { La técnica propuesta utiliza } \\
\text { tanto la información geométrica } \\
\text { (es decir, coordenadas XYZ) } \\
\text { como el color (es decir, RGB / } \\
\text { HSV), datos que son } \\
\text { proporcionados por un escáner } \\
\text { láser 3D y una cámara a color }\end{array}$ & $\begin{array}{l}\text { - detección de } \\
\text { puertas }\end{array}$ & \\
\hline $\begin{array}{l}\text { Vision-based adaptive } \\
\text { assistance and haptic } \\
\text { guidance for safe } \\
\text { wheelchair corridor } \\
\text { following [44] }\end{array}$ & $\begin{array}{l}\text { El objetivo de este proyecto es } \\
\text { colocar un sistema de } \\
\text { asistencia y guía adaptativo } \\
\text { para evitar colisiones con la } \\
\text { pared cuando un usuario } \\
\text { maneja manualmente una silla } \\
\text { de ruedas en un pasillo }\end{array}$ & $\begin{array}{l}\text {-control del } \\
\text { usuario y del } \\
\text { robot para el } \\
\text { manejo de } \\
\text { una silla de } \\
\text { ruedas a } \\
\text { través de } \\
\text { pasillos }\end{array}$ & \\
\hline $\begin{array}{l}\text { Arquitectura y diseño } \\
\text { de un sistema } \\
\text { completo de } \\
\text { navegación } \\
\text { semántica. } \\
\text { Descripción de su } \\
\text { ontología y gestión de } \\
\text { conocimiento [70] }\end{array}$ & $\begin{array}{l}\text { Esta tesis aborda la idea } \\
\text { subyacente de conseguir que } \\
\text { un robot se comporte de } \\
\text { manera semejante a como lo } \\
\text { haría un ser humano, } \\
\text { permitiéndole al robot una } \\
\text { mayor comprensión del } \\
\text { entorno y de los objetos que } \\
\text { contiene }\end{array}$ & $\begin{array}{l}\text { - Pasillos, los } \\
\text { trabaja como } \\
\text { figuras } \\
\text { geométricas } \\
\text { simples }\end{array}$ & $\begin{array}{l}\text { clasificación semántica del } \\
\text { entorno }\end{array}$ \\
\hline $\begin{array}{l}\text { El robot Moway, una } \\
\text { herramienta para el } \\
\text { aprendizaje basado en } \\
\text { proyectos [73] }\end{array}$ & $\begin{array}{l}\text { Proyecto basado proceso de } \\
\text { enseñanza-aprendizaje en ABP } \\
\text { para estudiantes de "Sistemas } \\
\text { Inteligentes" }\end{array}$ & $\begin{array}{l}\text { - Laberintos } \\
\text { (Pasillos y } \\
\text { Girar } \\
\text { esquina) }\end{array}$ & Planificación con incertidumbre \\
\hline $\begin{array}{l}\text { Topological } \\
\text { Autonomous } \\
\text { Navigation for Mobile } \\
\text { Robots in Indoor } \\
\text { environments using } \\
\text { Ann and FSM [30]. }\end{array}$ & $\begin{array}{l}\text { La aplicación del sistema } \\
\text { propuesto es un robot de } \\
\text { servicio de navegación en } \\
\text { interiores que puede moverse } \\
\text { de forma autónoma, designado } \\
\text { para la vigilancia y detección } \\
\text { de situaciones anormales (por }\end{array}$ & $\begin{array}{l}\text { - Seguir recto } \\
\text { - Girar a la } \\
\text { izquierda a la } \\
\text { derecha } \\
\text { - Inter- } \\
\text { secciones }\end{array}$ & $\begin{array}{l}\text { Se tiene que usar un ANN muy } \\
\text { robusto para tratar con } \\
\text { entradas que posean un alto } \\
\text { nivel de ruidos podrían } \\
\text { conducir a una clasificación } \\
\text { errónea y cambios de estado } \\
\text { inadecuadas, }\end{array}$ \\
\hline
\end{tabular}




\begin{tabular}{|c|c|c|c|}
\hline & $\begin{array}{l}\text { ejemplo, intrusiones, manchas } \\
\text { de fuego). }\end{array}$ & & \\
\hline $\begin{array}{l}\text { Control neuronal- } \\
\text { difuso de navegación } \\
\text { reactiva Continúa } \\
\text { incluyendo la evasión } \\
\text { de obstáculos } \\
\text { presentes y } \\
\text { emergentes [69]. }\end{array}$ & $\begin{array}{l}\text { En este trabajo se le permite al } \\
\text { robot que en ambientes } \\
\text { estructurados y } \\
\text { dinámicos, tenga la capacidad } \\
\text { de variar su velocidad de } \\
\text { desplazamiento, en virtud de la } \\
\text { composición del entorno } \\
\text { cercano y lejano, }\end{array}$ & $\begin{array}{l}\text { - Obstáculos } \\
\text { emergentes } \\
\text { - Puertas }\end{array}$ & $\begin{array}{l}\text { Las soluciones planteadas } \\
\text { requieren por obligación } \\
\text { ejecutarse en el entorno para } \\
\text { el cual fueron diseñados, en } \\
\text { caso de necesitar implementar } \\
\text { este tipo de sistemas en un } \\
\text { entorno distinto se presenta la } \\
\text { complejidad de tener que } \\
\text { implementar todo el sistema } \\
\text { guía y reprogramar al robot } \\
\text { para el nuevo esquema }\end{array}$ \\
\hline $\begin{array}{l}\text { Análisis de un } \\
\text { esquema de control } \\
\text { de comportamientos } \\
\text { basado en } \\
\text { subespacios nulos } \\
\text { para robots móviles } \\
\text { [45]. }\end{array}$ & $\begin{array}{l}\text { En este artículo se aborda el } \\
\text { problema de la navegación en } \\
\text { entornos no estructurados y } \\
\text { dinámicos, donde las tareas } \\
\text { asignadas se ordenan de } \\
\text { acuerdo a su prioridad y se } \\
\text { combinan de tal manera que } \\
\text { las de menor prioridad se } \\
\text { proyectan en el subespacio } \\
\text { nulo de las de mayor prioridad }\end{array}$ & $\begin{array}{l}\text { - Mínimos } \\
\text { locales }\end{array}$ & $\begin{array}{l}\text { Debido a que el método se } \\
\text { basa en funciones vectoriales, } \\
\text { se presenta el problema de los } \\
\text { mínimos locales }\end{array}$ \\
\hline $\begin{array}{l}\text { A visual servoing } \\
\text { approach for } \\
\text { autonomous corridor } \\
\text { following and doorway } \\
\text { passing a wheelchair } \\
{[14] .}\end{array}$ & $\begin{array}{l}\text { Trabajo desarrollado para } \\
\text { navegación autónoma en sillas } \\
\text { de ruedas. }\end{array}$ & $\begin{array}{l}\text { - Navegación } \\
\text { por pasillos } \\
\text { - Pasos de } \\
\text { Puerta }\end{array}$ & \\
\hline $\begin{array}{l}\text { Algoritmo de } \\
\text { navegación reactiva } \\
\text { de robots móviles para } \\
\text { tareas bajo } \\
\text { invernadero [16]. }\end{array}$ & $\begin{array}{l}\text { La aplicación de la plataforma } \\
\text { es para ser usada al interior de } \\
\text { los invernaderos, donde se } \\
\text { hace necesario pulverizar los } \\
\text { cultivos. }\end{array}$ & $\begin{array}{l}\text { Giros a } \\
\text { derecha e } \\
\text { izquierda } \\
\text { Navegación } \\
\text { por pasillos }\end{array}$ & $\begin{array}{l}\text { Un aspecto fundamental es el } \\
\text { proceso de giro, pues si dicho } \\
\text { proceso no es realizado de } \\
\text { forma correcta el robot podría } \\
\text { perderse o chocar contra las } \\
\text { plantas. } \\
\text { el algoritmo de navegación } \\
\text { implementado es totalmente } \\
\text { reactivo y no tiene en cuenta la } \\
\text { posición en la que se } \\
\text { encuentra el robot, no se } \\
\text { puede establecer un punto final } \\
\text { donde el robot pararía de } \\
\text { pulverizar }\end{array}$ \\
\hline $\begin{array}{l}\text { Contribución a la auto } \\
\text { localización de robots } \\
\text { móviles basada en la } \\
\text { fusión de información } \\
\text { multisensorial [79]. }\end{array}$ & $\begin{array}{l}\text { Trata sobre navegación en } \\
\text { Hitos. }\end{array}$ & -Esquinas & $\begin{array}{l}\text { Los procesos altamente no } \\
\text { lineales involucrados en la auto } \\
\text { localización de un vehículo } \\
\text { móvil son muy difíciles de } \\
\text { modelar }\end{array}$ \\
\hline
\end{tabular}




\section{RESULTADOS}

Muchos experimentos se han tenido que realizar para poder observar el comportamiento de las diversas técnicas existentes para comprobar sus pros y sus contras tanto en entornos simulados y en plataformas robóticas reales [22].

Varias técnicas se han tratado de usar en cada una de las fases que son necesarias para lograr el cumplimiento de una tarea, cada una de ellas ha mostrado suficiencias para algunas cosas pero inconvenientes para otras, por ello este es un campo en el cual hay mucho por trabajar e investigar.

Una correcta adquisición de los datos del entorno y un adecuado procesamiento de estos datos, les darán una buena oportunidad a los investigadores para adelantar diversos algoritmos de navegación y así poder cumplir con las diversas tareas que se presentan en la robótica móvil de una manera exitosa.

Después de todo el análisis realizado se presenta un resumen de algunos de los casos, donde se muestran presencia de los hitos mencionados, igualmente se muestra (ver tabla 1) cual es el enfoque que se da en cada uno de estos artículos y las técnicas utilizadas

\section{CONCLUSIONES}

En la robótica móvil con ruedas existen diferentes tipos de controladores para la navegación autónoma, donde algunos presentan mayor fortaleza en la estabilidad, otros en la velocidad, otros en la seguridad, pero ninguno ha tenido una total fiabilidad para todos los casos que se han proyectado, lo cual da un amplio espectro para seguir investigando en las diversas áreas.

Conforme el momento actual de los desarrollos en la robótica que pretenden ser aplicados a las diversas áreas de la academia y la industria, presentan algunas dificultades tales como costos y personas que los sepan manipular, hace difícil su aplicación fuera del ámbito de la investigación,

La adecuada escogencia de la sensorica con que se va a trabajar en las plataforma, le ayuda en gran medida a los Robots en cuanto a las posibles decisiones que este deba tomar, dado que una medida errónea o falsa implicaría una posible toma de determinaciones incorrectas

Dada la multiplicidad de métodos que existen para la planeación y generación de trayectorias en la robótica móvil con ruedas, es importante saber elegir que técnica se va a utilizar dependiendo de las características de la aplicación a desarrollar.

\section{LINEAS FUTURAS}

Se consideran las siguientes líneas futuras:

La integración de diversos tipos de algoritmos, los cuales permitan discriminar los diferentes tipos de objetos inmersos en cada ciclo de muestreo o en algunos casos hasta el tipo de superficie [9],

En el área de la navegación, donde previamente se le brinda al robot un mapa previo, como fábricas, hospitales y otros, sería deseable que se actualizara el mapa del entorno simultáneamente con la navegación, dado que se evitarían "falsos positivos" de los objetos del ambiente [70].

Dada la multiplicidad de tareas que pueda presentar un robot a la hora de cumplir una tarea se podrían paralelizar varios procedimientos empleando un procesador con varios núcleos o un procesador gráfico, esto para minimizar el tiempo de procesamiento [6].

En el área de apoyo y guía de sillas de ruedas se apunta a integrar la evasión local de obstáculos y la navegación a través del paso de puertas al sistema de control [44].

\section{AGRADECIMIENTOS}

El trabajo de Investigación al cual está asociada esta revisión, se ha desarrollado gracias a los aportes brindados por el Instituto Tecnológico Metropolitano y el Politécnico Colombiano Jaime Isaza Cadavid.

\section{BIBLIOGRAFÍA}

[1] Londoño, N. Metodologías de Desarrollo de Software, un enfoque a Robots Móviles. Revista Politécnica ISSN 1900-2351, Número 8, 74-83, (2009). 
[2] Pastor, J.; Rodríguez, F. J. La robótica como elemento de motivación del aprendizaje en los alumnos de ingeniería y potenciación de habilidades profesionales. 15 de julio 2015, de Departamento de Electrónica. Universidad de Alcalá España. Sitio web: http://espacio.uned.es:8080/fedora/get/taee:congreso2006-1040/S1K03.pdf.

[3] Lewis, F. Robotics". Mechanical Engineering Handbook. Frank Kreith. CRC Press LLC, 1999.

[4] Murphy, R.R. Introduction to Al Robotics. Editorial MIT Press. ISBN: 0-262-13383-0, 2000.

[5] Reale, F. P. Estudio del Estado del Arte en Robots Agrícolas. Universidad de la Republica de Uruguay, Uruguay, 2014.

[6] Montes, Martín. Sistema de navegación local para vehículo autónomo. Seminario de Investigación de invierno y segundo de divulgación de matemáticas aplicada, Vol. 1, 23-34. ISBN-13: 978-84-16399-97-0. 2016.

[7] T. Gomi, A. Gri_th, Developing intelligent wheelchairs for the handicapped, in: V. Mittal, H. Yanco, J. Aronis, R. Simpson (Eds.), Assistive Technology and Artificial Intelligence, Vol. 1458 of Lecture Notes in Computer Science, Springer Berlin Heidelberg, pp. 150178, 1998.

[8] S. P. Levine, D. A. Bell, L. A. Jaros, R. C. Simpson, Y. Koren, J. Borenstein, The navchair assistive wheelchair navigation system, IEEETransactions on Rehabilitation Engineering 7, pp 443-451, 1999.

[9] Cruz. Claudia \& Arellano. Jesús. Sistema de Navegación Reactiva Difusa para Giros Suaves de Plataformas Móviles Empleando el Kinect. Computación e Informática, Año 5 No. 3. Noviembre 2016.

[10] Sánchez, Luis Miguel. Desarrollo de una plataforma robótica móvil para búsqueda posterior a un sismo, Y Mitigación De Incendios De Baja Escala. Tesis de Maestría. Riobamba - Ecuador: Escuela Superior Politécnica De Chimborazo. 2017.

[11] Sales, Daniel Oliva; Shinzato, Patrick; Pessin, Gustavo; Osório, Fernando S. ;Wolf, Denis F,Vision-Based Autonomous Navigation System Using ANN and FSM Control. In: Proceedings of the IEEE LARS/EnRI, São Bernardo do Campo, SP. IEEE Society Press,. v.1. p. 85-90., 2011.

[12] Toapanda. Edison, Implementación de un sistema de navegación autónomo basado en
SLAM y navegación Reactiva. Trabajo de Pregrado. ESPE-Universidad de las fuerzas Armadas. Ecuador. 2018

[13] González Sánchez, Ramón et al, "Algoritmo de navegación reactiva de robots móviles para tareas bajo invernadero", XXVII Jornadas de Automática, ISBN: 84-689-9417-0, Almería, España, 2006.

[14] Babel, Marie \& Chaumette, Francois, A visual servoing approach for autonomous corridor following and doorway passing a wheelchair. Robotics and Autonomous Systems, 2014.

[15] Cordero, Martin \& Sepúlveda, Gabriel, Control de un robot móvil de ruedas mediante campos potenciales artificiales y procesamiento digital de imágenes en la evasión de obstáculos. Tesis de Maestría: Instituto Politécnico Nacional-México, 2011.

[16] González, R., "Desarrollo de algoritmos de navegación reactiva de robots móviles para tareas bajo invernadero", Proyecto Fin de Carrera, Universidad de Almería, 2006.

[17] F. Rodríguez, J. Sánchez-Hermosilla2 , L. Rivera1 , A. Giménez2. Análisis de viabilidad de la utilización de robots en invernaderos. Propuesta para el modelo Almería. II simposio nacional de ingeniería hortícola, Vol. 1, págs. 10-12. 2016.

[18] Cerrada, Carlos.Cerrada, José A. Valero, Enrique \& Abad, Ismael, Generación de comportamiento autónomo para un robot de inspección de cultivos. Centro de Automática y Robótica (CSIC-UPM),Universidad Nacional de Educación a Distancia (UNED), ISBN: 978-84695-8175-9, Madrid, España, 2013.

[19] Rodríguez Caro. Daniel,Martínez Tejada. Laura, Pardo Beainy. Camilo,Diseño e Implementación de un Prototipo de TGV Orientado a la Aplicación en Sistemas Telerrobóticos con Capacidad de Exploración y Mapeo, Facultad de Ingeniería Electrónica Universidad Santo Tomás Seccional Tunja, Vol.3, pp. 117-125, 2012.

[20] Mora Herrera. Héctor Tobías, Construcción de un Robot que preste servicios degasfitería, en ductos planos, semi inclinados, y subterráneos, Universidad Técnica Estatal De Quevedo, Ecuador, 2015.

[21] Silva Ortigoza. Ramon, Marcelino Aranda. Mariana\&Silva Ortigoza. Gilberto, Wheeled Mobile Robots: A review, IEEE Latin America Transactions, Vol. 10 pág.2209 - 2217, 2012. 
[22] Calisi, Daniele, Mobile robots and vehicles motion systems: a unifying framework, Tesis Doctoral, Universidad de Roma, Italia, 2009.

[23] Minguez J, Lamiraux F, Laumond JP, Motion planning and obstacle avoidance. In: Siciliano $\mathrm{B}$, Khatib $\mathrm{O}$ (eds) Springer Handbook of Robotics, Springer Berlin Heidelberg, 2008.

[24] Noreils, Fabrice\&Chatila, R., A general structure for mobile robot action control. IEEE/RSJ International Workshop on Intelligent and Systems. Tsukuba Japan, 1989.

[25] Ridao, P.; Batlle, J.; Amat, J.; Roberts, G.N., Recent trends in control architectures for autonomous underwater vehicles. International Journal of Systems Science. vol. 30. No 9. Págs 1033-1056, 1999.

[26] Brooks, Rodney, From Earwings to Humans. Robotic and Autonomous Systems. Vol 20. No 2.Págs. 291-304, 1997.

[27] Brooks, Rodney, Intelligence without Representation. Preprints of the Workshop in Foundations ofArtificial Intelligence. Endicott House. Dedham. MA, 1987.

[28] Suárez, A. F. \& Loaiza, H. Implementación de un esquema de navegación reactiva con sensores RGB-D. UIS Ingenierías, 14 (1), 719. 2015.

[29] Acosta. Gustavo, Gallardo. JoséyPérez. Ricardo. Arquitectura de control reactiva para la navegación autónoma de robots móviles. Ingeniare. Revista chilena de ingeniería, vol.24. 2016.

[30] Oliva, D., \& Al, e, Topological Autonomous Navigation for Mobile Robots in Indoor Environments using ANN and FSM. Universidad de San Pablo, Brazil, 2011.

[31] Ridao, P., Contreras, M.,Batlle, J.; Amat, J., O2CA2: A new Hibrid Control Architecture for a low cost AUV, Control Applicationin Marine Systems CAMS'2001 Scotland.

[32] Posadas, J.; Simo, J.; Poza, J, Una Arquitectura para el control de Robots móviles mediante delegación de código y sistemas Multiagente, IV Workshop en Agentesfísicos. Alicante-España, 2003.

[33] Vallejo Rodríguez, E.,Aprendizaje Evolutivo de Reglas Fuzzy en un sistema clasificador modificado para control de agentes móviles,Valencia, España, 2004.

[34] Kelly, A. et al. Mobile Robotics: Mathematics, Models, and Methods. New Yok, USA: Cambridge University Press. 2013.

[35] Carelli, Ricardo, Oliveira, Eduardo \& Freire, Corridor navigation and wall-following stable control for sonar-based mobile robots, Robotics and Autonomous Systems 45, págs. 235 - 247, 2003.

[36] Gallardo, Domingo, et al., Estimación bayesiana de características en robots móviles mediante muestreo de la densidad a posteriori, Departamento de Ciencia de la Computación e Inteligencia Artificial Universidad de Alicante, 1999.

[37] Isard, Michael and Blake, Andrew. Contour tracking by stochastic propagation of conditional density. In Proc. Europena Conf. Computer Vision 1996 (AAAl'98), pages 343356. Springer Verlag, 1996.

[38] Press, W.H., Teulosky, S.A., Vetterling, W.T. and Flannery, B.P. Numerical Recipes in C. Cambridge University Press, 1988.

[39] Isard, Michael and Blake, Andrew. Contour tracking by stochastic propagation of conditional density. In Proc. Europena Conf. Computer Vision 1996 (AAAl'98), pages 343356. Springer Verlag, 1996.

[40] Santos, Victor., Sandini, G., Curotto, F., Garibaldi, S., Divergent stereo in autonomous navigation: from bees to robots, International Journal of Computer Vision 14 159-177. 1995

[41] Carelli, R., Soria, C., Nasisi, O., Freire, E., Stable AGV corridor navigation with fused vision-based control signals, in: Proceedings of the 28th Conference of the IEEE Industrial Electronics Society, IECON, Sevilla, Spain, November págs. 5-8, 2002.

[42] Dev, A., Kröse, B., Groen, F., Navigation of a mobile robot on the temporal development of the optic flow, in: Proceedings of the IEEE/RSJ/GI International Conference on Intelligent Robots and Systems IROS'97, Grenoble, págs. 558-563, September 1997.

[43] Ollero, A., Planificación de trayectorias para Robots Móviles, Universidad de Málaga, 1995.

[44] Vishnu K. Narayanana, François Pasteaub , Maud Marchal c , AlexandreKrupa. Visionbased adaptive assistance and haptic guidance for safe wheelchair corridor following. Computer Vision and Image Understanding, vol. 149, pags 171-185. 2016.

[45] Acosta, Andrés, Hincapie, Roberto \& Acosta, Gustavo, Análisis de un esquema de control de comportamientos basado en subespacios nulos para robots móviles, ISSN 1657-7663. Revista Avances en sistemas e Informática, Vol 8, 165-172, 2011. 
[46] Antonelli, G. \&Arrichiello, F., Teh null-space based behavioral control. Intelligent Service Robotics, vol. 1, págs. 27- 39, 2008.

[47] Thrun, S., Probabilistic Robotics, Wolfram Burgard, Dieter Fox, 2000.

[48] Rodríguez, E., Diagramas de Voronoi, Cinvestav-Tamaulipas, 2010.

[49] MoralesElizabeth, Localización y mapeo simultáneo por robots móviles con ruedas basados en mapas de ocupación y matrices dispersas. Trabajo de Pregrado, Universidad Católica San Pablo: UCSP. 2018

[50] Luna, Karla Lourdes. Guía de Personas con Robots Móviles Basado en la Interacción Humano-Robot. Tesis de doctorado. Universidad Autónoma de San Luis Potosí Facultad de Ingeniería: San Luis Potosí. 2018.

[51] Freire, Eduardo \& Soria, Carlos. Stable agv corridor navigation with fused redundant control signals. Instituto de Automática Universidad Nacional de San Juan, Argentina, VII SBAI/ II IEEE LARS, 1-8, 2005.

[52] T. H. Yuan, F. H. Hashim, W. M. D. W. Zaki, and A. B. Huddin, "An Automated 3D Scanning Algorithm using Depth Cameras for Door Detection". 2015 International Electronics Symposium (IES) pp. 58-61, 2015.

[53] D. Anguelov, D. Koller, E. Parker, and S. Thrun, "Detecting and modeling doors with mobile robots," IEEE Int. Conf. Robot. Autom. 2004. Proceedings. ICRA '04. 2004, vol. 4, 2004.

[54] Y. Priyadarshana and G.imalaratne, "Sensing environment through mobile: $A$ personalizedwearable obstacle detection system for visually impaired people," International Journal of Soft Computing and Engineering, vol. 3, no. 6, pp. 81-88, Jan. 2014.

[55] B. Quintana, S. A. Prieto, A. Adán. Door Detection in 3D Colored Laser Scans for Autonomous Indoor Navigation 2016 International Conference on Indoor Positioning and Indoor Navigation (IPIN), pags 4-7, Alcalá de Henares, Spain. October 2016

[56] T. H. Yuan, F. H. Hashim, W. M. D. W. Zaki, and A. B. Huddin, "An Automated 3D Scanning Algorithm using Depth Cameras for Door Detection". 2015 International Electronics Symposium (IES) pp. 58-61, 2015.

[57] M. M. Shalaby, M. A. M. Salem, A. Khamis, and F. Melgani, "Geometric model for vision-based door detection," Proceedings of 2014 9th IEEE International Conference on Computer
Engineering and Systems, ICCES 2014, pp. 41-46, 2015.

[58] Garcia, Manuel. Localización y cartografíasimultanea: estudio sobre la exploraciónoptima de escenarios complejos. Universidad de Guanajuato: Salamanca España. 2017.

[59] G. Dudek and M. Genkin. Computational principles of mobile robotics. New York, USA. Cambridge University Press. 2nd ed. 2010

[60] Marino, A., Parker, L.,Antonelli, G. and Caccavale, F., Behavioral Control for MultiRobot Perimeter Patrol: A Finite State Automata approach. In: ICRA, 2009.

[61] Yerubandi, V., Reddy, Y. M., \& Kumar, M. V. Navigation system for an autonomous robot using fuzzy logic.International Journal of Scientific and Research Publications, Volume 5, Issue 2, February. 2015.

[62] Ojeda, Lauro \& Borenstein Johann, FLEXnav: Fuzzy logic Expert Rule-based Position Estimation for Mobile Robot on Rugged Terrain. Proceedings of the 2002 IEEE International conference on Robotics and Automation. Washington DC, USA, pags 317322, 2002.

[63] Negenborn, Rudy, Robot Localization and Kalman Filter, 2003.

[64] Smith, R., Self, M. \& Cheeseman, P., A Stochastic mapfor uncertain spatial relationships, Workshop on spatial Reasoning and Multisensor Fusion, 1987.

[65] Atkeson, Chis, An extended Kalman Filter for a mobile Robot, 2004.

[66] W. Yuan, Z. Li and C.Y. Su, RGB-D sensorbasedvisual SLAM for localization and navigation of indoor mobile robot. In 2016 International Conference on Advanced Robotics and Mechatronics (ICARM), Págs. 82-87. 2016

[67] S. Jain, S. Nandy, R. Ray, and S. Shome, Application of particle filtering technique for sensor fusionin mobile robotics. In Mechatronics and Automation (ICMA), 2011 International conference on, págs. 2285-2290. 2011

[68] Thrun, Sebastian, Probabilistic Algorithms in Robotics. Al MagazineAAAI, vol. 21 núm. 4, págs. 93.109, 2000.

[69] Correa, Felipe, Control Neuronal-Difuso de navegación reactiva continúa incluyendo la evasión de obstáculos presentes y 
emergentes, Antofagasta, Chile: Universidad Católica Del Norte, 2013.

[70] Crespo. Jonathan, Arquitectura y diseño de unsistema completo de navegaciónsemántica. Descripción de su ontología y gestión de conocimiento. Tesis Doctoral. Universidad Carlos III de Madrid. 2017.

[71] Parrón, F., Gonzáles, R. \& Rodríguez, F., Sistema de localización para Robots móviles basado en GPS. Aplicación a campos de Golf, CEA jornadas de Automática. Valladolid, España, 2009.

[72]Ortega, L., El Diagrama de Voronoi, Universidad de Jaen, España, 2010.

[73] Pagola. Miguel et al. El robot Moway, una herramienta para el aprendizaje basado en proyectos. Actas de las XXII Jenui. Almería, ISBN: 978-84-16642-30-4 Páginas: 43-49. 6-8 de julio 2016

[74] Miranda Cruz, Luis, Humanoid Robot NAO: Developing Behaviours for Soccer Humanoid Robots. Master in Informatics and Computing Engineering, Facultad de Ingeniería de Universidad de Porto, 2011.

[75] Muñoz Ceballos, Nelson David, Londoño Ospina, Nelson De Jesús, Ortiz, Luis Fernando, Método de comparación de algoritmos de control de robots móviles, Memorias Congreso $\mathrm{CISCl}$, Miami-Estados Unidos, 2008.

[76] Rosenblatt, j.\& William, S., Behavior Based Control for Autonomous Exploration, IEEE, 2006.

[77] Yandún, Aracely \& Sotomayor, Nelson, Planeación y seguimiento de trayectorias para un robot móvil, Escuela Politécnica Nacional, Quito - Ecuador, 2012.

[78] Budiharto, W., Santoso, A., Purwanto, D. and Jazidie, A. Multiple Moving Obstacles Avoidance of Service Robot using Stereo Vision. TELKOMNIKA, 9(3), 433 444. 2011.

[79] Navarro, Danilo Alfonzo. Contribución a la autolocalización de robots móviles basada en la fusión de información multisensorial. Tesis Doctoral. Universidad Politécnica de Valencia, España, 2009

[80] Patentes DE3827619 y DE3827620, "Sensorcontrolled tracking device, especially for levelling of ground surfaces", 1990.

[81] Patente US2004112662, "Contact detecting device and vehicle mounting the same", 2004.

[82] Patente US7133123, "Laser obstacle avoidance apparatus", 2006.
[83] Patente ES2322220B1, "Dispositivo útil para la generación de mapas de contorno y sus aplicaciones", 2007

[84] Gil, Alejandro y otros. Construcción del plano de entornos de trabajo cerrados utilizando el robot Koala". Pistas Educativas, No. 125, México, Tecnológico Nacional de México en Celaya. Págs. 190-205. Octubre 2017,

[85] Nakhaeinia, D. and Karasfi, B. A behaviorbased approach for collision avoidance of mobile robots in unknown and dynamic environments. Journal of Intelligent \& Fuzzy Systems (24), 299-311. 2013. 